\title{
Aging may Affect Insulin Action Independent of Adiposity in Non- Diabetic Subjects
}

\author{
Yoshihisa Okamoto ${ }^{1}$, Tadashi Yamakawa ${ }^{1}$ and Yasuo Terauchi² \\ ${ }^{1}$ Department of Endocrinology and Diabetes, Yokohama City University Medical Center, Yokohama, Japan \\ ${ }^{2}$ Department of Endocrinology and Metabolism, Graduate School of Medicine, Yokohama City University, Yokohama, Japan
}

\section{See article vol. 23: 207-215}

Obesity, particularly with visceral fat accumulation (visceral obesity), accelerates the cluster of metabolic disorders and finally results in atherosclerotic cardiovascular diseases that are recognized as metabolic syndrome $(\mathrm{Met} S)^{1)}$. Aging, a physiological change that is initiated at birth, can affect various metabolisms, including glucose tolerance mediated by insulin action such as insulin sensitivity and secretion. In Japan, patients over 65 years of age account for two-thirds of all medical expenses for diabetes mellitus (DM) ${ }^{2}$. Therefore, the prevention and treatment of age-related DM are major healthcare issues in the country. However, it is unclear whether and how aging affects insulin action and whether the effect is dependent on or independent of (visceral or subcutaneous) fat accumulation and distribution.

A report by Hirose, et al. ${ }^{3)}$ may provide an insight on this issue. They analyzed Japanese male and female participants aged 35-84 years in a 2-year comprehensive health checkup program at their institute. They excluded participants who were on medication for $\mathrm{DM}$ or who met the pre-determined exclusion criteria. The remaining healthy subjects were divided into five age groups (by 10-year increments) to analyze the effect of aging on factors such as the clinical parameters for fat accumulation and distribution and insulin action. Aging was associated with a linear increase in visceral and subcutaneous fat areas (VFA and SFA) and VFA/SFA ratios in both male and female participants, as expected. These results indicated that age promoted the dominant accumulation of visceral fat.

Address for correspondence: Yoshihisa Okamoto, Department of Endocrinology and Diabetes, Yokohama City University Medical Center, 4-57 Urafune-cho, Minami-ku, Yokohama,

Kanagawa, 232-0024, Japan

E-mail: yokamoto@yokohama-cu.ac.jp

Received: November 6, 2015

Accepted for publication: November 7, 2015
These findings are reasonable because elderly subjects have reduced physical activity, and they use less body energy compared with younger subjects. Moreover, several hormones that affect the quantity and quality of fat, e.g., growth hormone/insulin-like growth factor- 1 and sex steroids, change with age ${ }^{4)}$.

Next, the authors evaluated the effects of aging on glucose tolerance parameters. Age was significantly negatively correlated with immunoreactive insulin (IRI), homeostasis model assessment of insulin resistance (HOMA-IR), and $\beta$-cell function (HOMA- $\beta$ ). In this study, HOMA-IR demonstrated weak but significant negative correlations with VFA and VFA/SFA in both males and females; however, these relationships disappeared after adjusting for age. Although the contribution of visceral fat or BMI on insulin resistance has been controversial ${ }^{5,6}$, we did not expect these results, wherein age may have influenced insulin action independent of fat accumulation or distribution.

Adiponectin (APN) is an adipocytokine that enhances insulin sensitivity, and a reduced plasma level of this protein in subjects with visceral obesity reduces their insulin resistance ${ }^{7)}$. A previous report revealed that centenarians who were more sensitive to insulin and less likely to have DM maintained higher APN plasma levels ${ }^{8}$. The authors measured high molecular weight (HMW)-APN, a beneficial form of APN, in a small number of participants. HMW-APN was negatively correlated with HOMA-IR and positively correlated with age. Importantly, HMW-APN demonstrated a much stronger negative correlation with HOMA-IR than with VFA. Thus, aging may be associated with higher plasma levels of HMW-APN, and this should ameliorate insulin resistance and reduce the necessary insulin amount, which is independent of VFA.

HOMA-IR or HOMA- $\beta$ is an index calculated by fasting IRI and blood glucose. When fasting blood glucose does not change, as in this study, the reduction of IRI directly reflects the decrease of HOMA-IR 


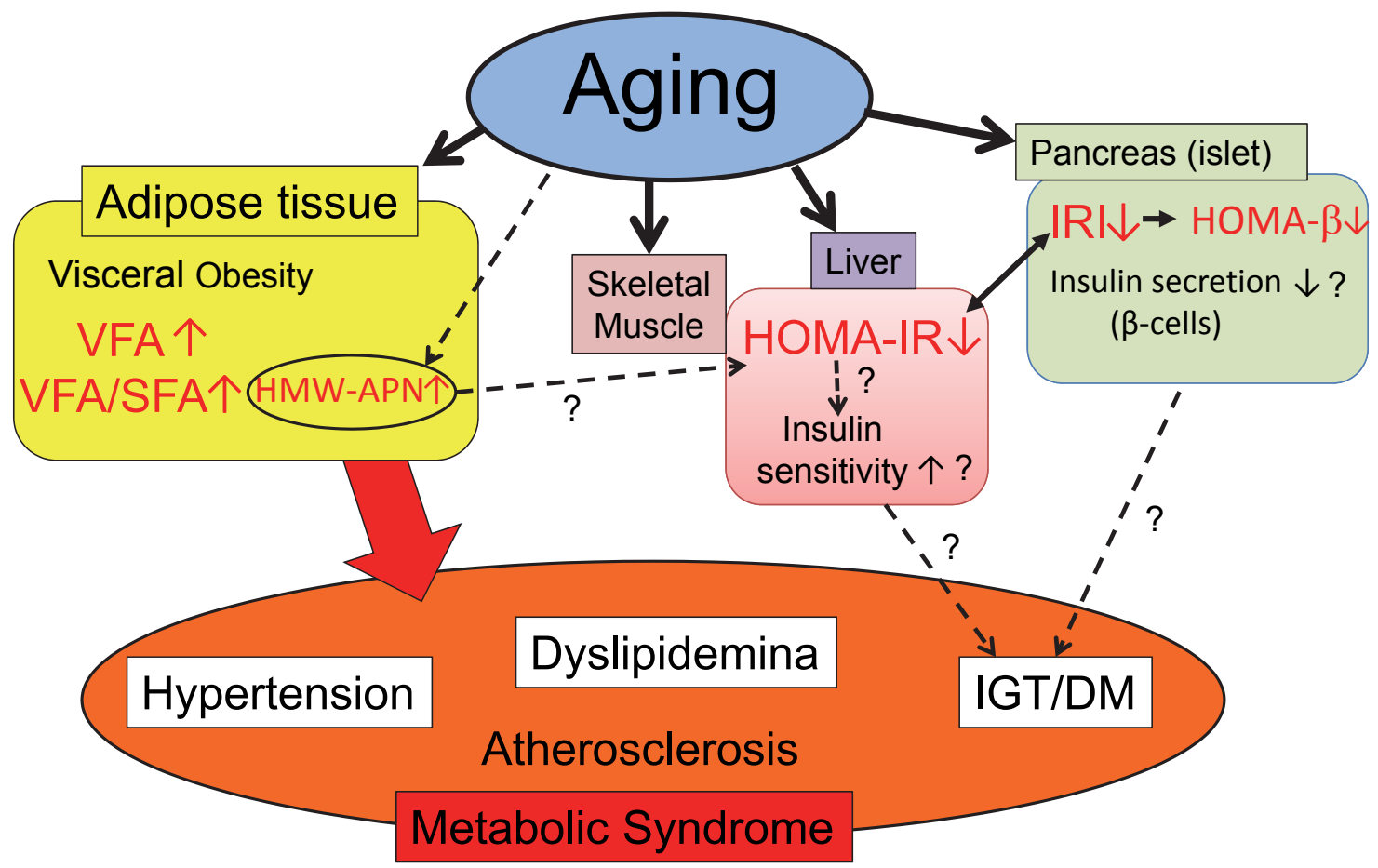

Fig. 1. The scheme of authors' theory.

Aging increases VFA and VFA/SFA ratios as well as plasma HMW-APN. On the other hand, aging decreases IRI, HOMA-IR, and HOMA- $\beta$ independent of fat accumulation or distribution (VFA, VFA/SFA). The results highlighted in red are found in this study.

and HOMA- $\beta$, thereby indicating increased insulin sensitivity and decreased insulin secretion capacity, respectively. On the basis of the overall findings, we can suggest two hypotheses that are independent of fat amount or distribution. 1) Aging ameliorates insulin resistance by reducing hyperinsulinemia, partially through HMW-APN. 2) Aging causes $\beta$-cell dysfunction (apoptosis), which reduces insulin secretion (HOMA- $\beta$ ) and subsequently lowers HOMA-IR. However, no cause and effect relationship was observed in this study. Thus, a longitudinal study or other clinical assessment for insulin resistance, such as the euglycemic insulin clamp technique, can answer this question, as the authors mentioned.

Senescence, the physiological deterioration of cellular function by aging, in adipose tissue has been proposed as a potential mechanism in obesity-related DM or Met $S^{9)}$. Furthermore, sarcopenia, the degenerative loss of skeletal muscle mass, strength, and function by aging, is a physiological condition prone to cause insulin resistance among the elderly ${ }^{10)}$. These aging factors must be taken into account when discussing the effect of aging on insulin resistance. Moreover, analyses in patients with DM may show different results because their biological contribution of visceral fat may be greater. However, the current report by Hirose et al. ${ }^{3)}$ proposed an interesting theory that aging can be an independent factor affecting IRI, HOMA-IR, and HOMA- $\beta$, despite it promoting visceral fat accumulation among healthy or health-conscious subjects (Fig. 1). This report may provide some information to understand the physiological mechanism of age-related glucose tolerance.

\section{Conflicts of Interest}

None.

\section{References}

1) Matsuzawa $Y$, Funahashi $T$ and Nakamura $T$ : The concept of metabolic syndrome: contribution of visceral fat accumulation and its molecular mechanism. J Atheroscler Thromb, 2011; 18: 629-639

2) Estimates of National Medical Care Expenditure 2014 Statistical Surveys conducted by Ministry of Health Labor and Welfare (Japan),

3) Hirose $H$, Takayama M, Iwao $Y$ and Kawabe $H$ : Effects of Aging on Visceral and Subcutaneous Fat Areas and on 
Homeostasis Model Assessment of Insulin Resistance and Insulin Secretion Capacity in a Comprehensive Health Checkup. J Atheroscler Thromb, 2016; 23: 207-215

4) Barzilai N, Huffman DM, Muzumdar RH and Bartke A: The critical role of metabolic pathways in aging. Diabetes, 2012; 61: 1315-1322

5) Gabriely I, Ma XH, Yang XM, Atzmon G, Rajala MW, Berg AH, Scherer P, Rossetti L and Barzilai N: Removal of visceral fat prevents insulin resistance and glucose intolerance of aging: an adipokine-mediated process? Diabetes, 2002; 51: 2951-2958

6) Ferrannini E, Vichi S, Beck-Nielsen H, Laakso M, Paolisso G and Smith U: Insulin action and age. European Group for the Study of Insulin Resistance (EGIR). Diabetes, 1996; 45: 947-953
7) Okamoto Y, Kihara S, Funahashi T, Matsuzawa Y and Libby P: Adiponectin: a key adipocytokine in metabolic syndrome. Clinical science (London, England: 1979), 2006; 110: 267-278

8) Arai Y, Takayama M, Abe Y and Hirose N: Adipokines and aging. J Atheroscler Thromb, 2011; 18: 545-550

9) Minamino T, Orimo M, Shimizu I, Kunieda T, Yokoyama M, Ito T, Nojima A, Nabetani A, Oike Y, Matsubara H, Ishikawa $\mathrm{F}$ and Komuro I: A crucial role for adipose tissue p53 in the regulation of insulin resistance. Nat Med, 2009; 15: 1082-1087

10) Ishii $S$, Tanaka $T$, Akishita $M$, Ouchi $Y$, Tuji T, Iijima $K$ and Kashiwa study i: Metabolic syndrome, sarcopenia and role of sex and age: cross-sectional analysis of Kashiwa cohort study. PLoS One, 2014; 9: e1127182 